\title{
Conducta autolesiva no suicida en adolescentes y su relación con factores personales y contextuales
}

\author{
Diana Obando, Ángela Trujillo y Manuel Prada \\ Universidad de La Sabana, Chía, Colombia
}

\begin{abstract}
Non-suicidal self-injury among adolescents and its relationship with personal and contextual factors. Epidemiological studies in mental health indicate an increase in the prevalence of non-suicidal self-injury behaviors in adolescents. However, few studies in Latin America have explored this problematic behavior in depth. Through two studies using a mixed methodology, this work consisted in characterizing self-injury and in identifying associated clinical variables in a sample of 1,517 adolescents and 43 patients. Additionally, the mediator effect of sensation seeking in the relationship between family attachment and self-injury was assessed. The quantitative results indicate a relationship between self-injury and personal and contextual variables such as impulsivity, sensation seeking and family dynamics. Qualitative analysis indicates that self-injury was present in comorbidity with other complex problematics at the affective, social, academic and familiar levels of the participants. The relevance of the results is discussed as to clinical context.
\end{abstract}

Keywords: Non-suicidal self-injury; adolescence; family dynamics; sensation seeking; impulsivity.

Resumen: Diferentes estudios epidemiológicos en salud mental indican un aumento en la prevalencia de los comportamientos autolesivos en adolescentes. Sin embargo, escasos estudios en Latinoamérica han examinado esta problemática en profundidad. Basándose en dos estudios con metodología mixta, el objetivo del presente trabajo consistió en la caracterización de la autolesión y en la identificación de variables clínicas asociadas a una muestra de 1.517 adolescentes y de 43 pacientes. Así mismo, se evaluó el efecto mediador de la búsqueda de sensaciones en la relación entre el apego familiar y la autolesión. Los resultados cuantitativos indican una relación entre la autolesión y los factores personales y contextuales, tales como la impulsividad, la búsqueda de sensaciones y las dinámicas familiares. El análisis cualitativo indica que la autolesión se presenta en comorbilidad con otras problemáticas complejas en los niveles afectivo, social, académico y familiar de los pacientes. Se discute la relevancia de los resultados en el ámbito clínico.

Palabras clave: Conducta autolesiva no suicida; adolescencia; dinámica familiar; búsqueda de sensaciones; impulsividad.

\section{Introducción}

Cada cuarenta segundos ocurre un suicidio en el mundo. En consecuencia, este problema ha sido considerado como la segunda causa de mortalidad en personas entre los 15 y 29 años (Organización Mundial de la Salud, 2014). En América Latina se reportaron 65.000

Recibido: 07 de febrero 2018; aceptado: 10 de mayo 2018 Correspondencia: Manuel Prada, Facultad de Psicología, Universidad de La Sabana, Campus Puente del Común Kilometro 7 Autonorte Bogotá, Chía, 140013 Cundinamarca, Colombia. Correo-e: manuel.prada1@unisabana.edu.co casos de suicidio entre los años 2005 y 2009 , de los cuales el $6.8 \%$ correspondían a edades entre los 10 y 19 años (Organización Panamericana de la Salud, 2014). Particularmente, en el año 2015 en Colombia se reportaron 2068 casos de suicidio, de los cuales el 10.6\% fueron de jóvenes entre los 10 y los 17 años (Instituto Nacional de Medicina Legal y Ciencias Forenses, 2016).

Estudios previos han identificado una estrecha relación entre el suicidio y la conducta autolesiva. Esta última se ha definido como un comportamiento directo y deliberado en el cual la persona se autoinflige daño o dolor en la superficie corporal por medio de cortes, quemaduras, rasguños y otros comportamientos, sin una in- 
tención suicida (Klonsky, 2007; Nock y Favazza, 2009). Sin embargo, la autolesión se ha identificado como un factor de riesgo que aumenta hasta en un 50\% la probabilidad de cometer suicidio (Beckman et al., 2016).

Estudios globales acerca de la prevalencia de este comportamiento indican que se manifiesta con más frecuencia en adolescentes (Brunner et al., 2014; Lin et al., 2017; Muehlenkamp, Claes, Havertape, y Plener, 2012; Thyssen y Van Camp, 2014). De acuerdo con el Estudio de Carga de Enfermedad Global (Global Burden of Disease Study, 2010), la autolesión constituye la causa número 18 de mortalidad, con un incremento de casos del $115 \%$ en los últimos 20 años. Sin embargo, a pesar de su relevancia, no hemos identificado suficientes estudios acerca de las características y la prevalencia de la conducta autolesiva no suicida en adolescentes Latinoamericanos.

Los estudios revisados hasta el año 2018 se enfocan en la identificación de los factores de riesgo asociados con la autolesión y en la función que cumple la autolesión en quienes la presentan. Al respecto, los resultados apuntan a que la autolesión es más frecuente en población femenina (Sornberger, Heath, Toste, y McLouth, 2012), con una edad de inicio entre los 13 y 15 años (Zetterqvist, Lundh, y Svedin, 2013). Acerca de la función, estudios previos sugieren que la autolesión se mantiene por múltiples fuentes de refuerzo con una alta variabilidad entre los sujetos que la presentan. Entre las funciones más frecuentes se encuentran la autoestimulación, la regulación emocional, el autocastigo, la búsqueda o distanciamiento social y la disminución de síntomas como la disociación o los deseos suicidas (Klonsky, 2007). Estas variaciones han sido explicadas desde los conceptos conductuales de refuerzo positivo y negativo, tanto a nivel personal como social (Robertson, Miskey, Mitchell, y Nelson-Gray, 2013).

Así mismo, diversos factores psicosociales se han relacionado con la autolesión. Entre los más frecuentes se encuentran los altos niveles de estrés (Baetens et al., 2014), las prácticas parentales negativas (Di Pierro, Sarno, Perego, Gallucci, y Madeddu, 2012) y el abuso físico, psicológico y sexual en la infancia (Baetens et al., 2014). Así mismo, existe una alta comorbilidad entre la autolesión y otras problemáticas psicológicas tales como el bajo estado de ánimo (Wilcox et al., 2012), los comportamientos agresivos (Tang et al., 2013), el déficit en habilidades de regulación emocional (Lin et al., 2017), la ansiedad (Ross y Heath, 2002), así como con síntomas de trastornos de la conducta alimentaria (Hilt, Nock, Lloyd-Richardson, y Prinstein, 2008), el consumo de sustancias psicoactivas (Spears, Montgomery, Gunnell, y Araya, 2014) y las conductas externalizantes (Adrian, Zeman, Erdley, Lisa, y Sim, 2011).
De manera específica se ha identificado que la impulsividad en los adolescentes aumenta la presencia de conductas de riesgo, entre ellas la autolesión (Cyders, Coskunpinar, y VanderVeen, 2016), puesto que se caracteriza por ser una acción repentina generada por un estado de ánimo negativo y sin el reconocimiento de las consecuencias que esta conducta puede suponer (Bresin, Carter, y Gordon, 2013; Peters, Baetz, Marwaha, Balbuena, y Bowen, 2016). Otro factor de riesgo asociado a esta problemática es el déficit en habilidades sociales ya que los conflictos y eventos interpersonales negativos que resultan estresantes para el adolescente aumentan la probabilidad de desplegar conductas autolesivas (Adrian et al., 2011).

Finalmente, la búsqueda de sensaciones es otro factor personal que se ha relacionado con la autolesión. Dicha conducta se orienta a la exploración de experiencias intensas con el objetivo de tomar riesgos físicos, sociales, económicos o de otra índole (Zuckerman, 1994). Trujillo, Obando, y Trujillo (2016) sostienen que las dinámicas familiares influyen en la exhibición de este tipo de comportamientos exploratorios. Particularmente, los conflictos familiares, el bajo control parental y la afectividad negativa tienen un efecto en el aumento de conductas autodestructivas en adolescentes (Martin et al., 2016).

\section{Estudio 1}

De acuerdo con los estudios previos revisados y teniendo en cuenta el limitado número de investigaciones en el contexto latinoamericano acerca de esta problemática, el Estudio 1 tuvo por objetivo describir la prevalencia de conductas autolesivas, los métodos implementados y su función en una muestra no clínica de adolescentes colombianos. Así mismo, el estudio se dirigió a identificar la relación entre la autolesión y factores personales (impulsividad, búsqueda de sensaciones y habilidades sociales) y contextuales (apego, conflicto y pobre manejo familiar) que previamente han sido asociados con la exhibición de esta conducta. Finalmente, por medio del Estudio 1 se buscó identificar si los factores personales constituyen variables mediadoras en la presunta relación entre la dinámica familiar y el comportamiento autolesivo en la muestra de estudio.

Nuestras hipótesis fueron las siguientes: (1) la autolesión se correlaciona de forma significativa tanto con factores personales como familiares; y (2) los factores personales median la relación existente entre el apego familiar y el comportamiento autolesivo. 


\section{Método}

\section{Participantes}

Este estudio contó con la participación de 1.517 adolescentes, estudiantes de colegios al norte de Bogotá, con una edad media de 14.7 años (rango $=11$-18 años; $D T=2.02$ ). La muestra incluyó la participación de chicos y chicas en porcentajes similares. Como criterio de exclusión se tuvo en cuenta que los estudiantes no quisieran participar de forma voluntaria o que los padres o cuidadores no autorizaran su participación por medio del consentimiento informado.

\section{Procedimiento}

La primera fase del estudio se centró en la consecución de la muestra por medio del contacto con instituciones educativas del sector norte de Bogotá, a quienes se les presentó el proyecto para que accedieran a socializarlo con las familias con el fin de obtener su consentimiento. Los padres que estuvieron de acuerdo con la participación de sus hijos firmaron el consentimiento informado en el cual se especificaba la confidencialidad de los datos recogidos y la voluntariedad de la participación. La aplicación de los instrumentos a los adolescentes tuvo una duración promedio de una hora y se llevó a cabo en las instalaciones de los colegios de manera grupal con el apoyo de un equipo de evaluadores previamente entrenado.

\section{Instrumentos}

Inventario de Afirmaciones acerca de la Conducta Autolesiva (ISAS; Inventroy of Statements About Self-Injury; Klonsky y Glenn, 2009; versión en español de Silva et al., 2016). Este inventario de autoinforme evalúa la presencia de conductas autolesivas de forma deliberada y sin intención suicida, así como las funciones que tienen estos comportamientos en adolescentes. El instrumento está compuesto por dos apartados: el primero llamado comportamientos que contiene 7 preguntas acerca de la presencia, tipo, frecuencia e intensidad de la autolesión; cuando el adolescente ha marcado uno o varios de estos comportamientos se le pide que responda el segundo apartado llamado funciones; éste consta de 39 afirmaciones que se responden en una escala de 3 puntos $(0=$ no relevante, $1=$ algo relevante, $2=$ muy relevante $)$. Los puntajes para cada una de las funciones puede variar de 0 a 6 y permiten identificar la función que tiene la conducta autolesiva, por ejemplo «cuando me autolesiono busco calmarme» y «cuando me autolesiono busco encajar con otros». Para el presente estudio se tuvo en cuenta la cantidad y el tipo de comportamientos autolesivos y su función. La prueba original tiene una consistencia interna de .84 para el apartado de comportamientos (Klonsky y Olino, 2008) y de .88 para el apartado de funciones (Klonsky y Glenn, 2009). De manera similar en esta muestra el alfa de Cronbach fue de .83 para el apartado de comportamientos y .87 para el de funciones.

Escala de Habilidades Sociales Messy (MESSY; Matson Evaluation of Social Skills with Youngers; Matson, Rotatori, y Helsel, 1983; version en español de Trianes et al., 2002). Esta escala evalúa habilidades sociales específicas en relación con la conducta social adaptativa y no adaptativa de niños y jóvenes entre los 4 y los 18 años. Consta de 5 subescalas (habilidades sociales apropiadas, asertividad inapropiada, impulsividad, sobre confianza y celos/soledad) las cuales se obtienen a partir de 61 ítems que se responden en una escala tipo Likert de 4 puntos (1 = nunca, $2=$ a veces, $3=$ a menudo, $4=$ siempre). Una mayor puntuación indica niveles más altos comportamiento inadecuados para las dimensiones de comportamientos negativos, o adecuados para las dimensiones de comportamientos positivos. La prueba origninal tiene una consistencia interna que varía entre .54 y .93 (Matson et al., 2010). Para el presente estudio se aplicaron los ítems correspondientes a la sub-escala de habilidades sociales apropiadas (25 ítems) debido a los intereses de los investigadores, la cual mide conductas como expresividad emocional, tener amigos, entre otras, con preguntas como «miro a los ojos a la gente cuando hablo con ella». La escala de habilidaes apropiadas ha sido validada en países suramericanos (Ipiña, Molina, y Reyna, 2010) con una consistencia interna adecuada de .81. Para el presente estudio, la escala presentó una consistencia interna de .92 .

Escala de Impulsividad de Barratt en Adolescentes (BIS-11; Patton y Stanford, 1995; versión en español de Salvo y Castro, 2013). Esta escala de autoreporte evalúa la impulsividad a través de tres subescalas: cognitiva, con preguntas como «mis pensamientos pueden tener gran velocidad»; motora, «digo las cosas sin pensarlas»; y no planeada «planifico mis actividades con antelación». Así mismo, permite obtener una puntuación global, donde puntajes altos indican una mayor impulsivdad. Consta de 30 ítems con una escala tipo Likert de 4 puntos $(0=$ raramente $o$ nunca, 1 =ocasionalmente, $3=$ a menudo, $4=$ siempre o casi siempre). La preuba original tiene una consistencia interna de .76 de acuerdo con los autores. Para la presente investigación, la prueba presentó un ínidice alfa de Cronbach adecuado (.64) para el total de la prueba.

Communities That Care -Youth Survey (CTC-YS; Arthur, Hawkins, Pollard, Catalano, y Baglioni, 2002). Este cuestionario permite identificar los niveles de exposición a factores de riesgo y protección asociados con el consu- 
mo de sustancias y conductas problemáticas en adolescentes, a través de 34 factores que se calculan a partir de los 165 ítems, con una consistencia interna entre $.59 \mathrm{y}$ .88. Para el presente estudio se incluyeron los factores familiares de conflicto, pobre manejo y apego, y a nivel personal el factor búsqueda de sensaciones. El conflicto familiar se evaluó en una escala de 4 puntos $(1=$ definitivamente si, $2=$ si, $3=$ no, $4=$ definitivamente no) que incluye preguntas como «las personas en mi familia se insultan o se gritan entre ellas». El pobre manejo familiar se evaluó con la misma escala, con ítems como «cuando no estoy en casa alguno de mis padres sabe dónde estoy y con quien». El factor protector familiar de apego utiliza la misma escala e incluye frases como «comparto mis pensamientos y sentimientos con mi madre». El factor búsqueda de sensaciones se evaluó por medio de una escala de 6 puntos $(1=$ nunca lo he hecho, $2=$ si, pero el año pasado, $3=$ menos de una vez al mes, $4=$ una vez al mes, $5=$ dos o tres veces al mes, $6=$ una vez a la semana o más) con ítems como: «he hecho lo que se siente bien sin que nada más importe». Un puntaje alto en cada factor indica mayores niveles de riesgo o mayores niveles de protección según corresponda. Este instrumento ha sido implementado en Colombia, con una consistencia interna adecuada (Trujillo et al., 2016). Para la presente investigación los cuatro factores seleccionados presentaron una consistencia interna entre .60 y .73 .

\section{Análisis estadístico}

El análisis de los datos se llevó a cabo por medio del paquete estadístico SPSS versión 24, calculándose la frecuencia de la autolesión, los métodos implementados y la función de la misma. A continuación se calcularon las correlaciones de Pearson entre las variables de estudio. Finalmente, se probó un modelo de mediación simple siguiendo el procedimiento recomendado por Hayes (2017) para identificar el efecto del apego familiar en la conducta autolesiva a través de la mediación de la bús- queda de sensaciones. En concreto, por medio de regresiones lineales se determinó el efecto del apego familiar sobre la variable mediadora (búsqueda de sensaciones), así como su efecto sobre la conducta autolesiva, controlando el efecto del sexo y la edad. El modelo se calculó utilizando el plug in de PROCESS (Hayes, 2017) en el paquete estadístico de SPSS.

\section{Resultados}

Los resultados que se derivan del análisis de frecuencias indican que el $46 \%$ de la muestra ha presentado algún tipo de conducta autolesiva. De este porcentaje se identificó que los métodos de autolesión más usados son los pellizcos $(27 \%)$, mordiscos $(23.4 \%)$, cortes $(22.1 \%)$, golpes $(17 \%)$, rasguños $(14.4 \%)$, arrancarse el pelo $(13.3 \%)$, chuzarse (insertar agujas en la superficie corporal; $13 \%)$, quemaduras $(11 \%)$, raspones en superficies $(9 \%)$ y escarbarse la piel ( $9 \%)$.

Los resultados también permiten determinar la cantidad de métodos que los adolescentes usan para autolesionarse. Al respecto, se identificó que el $32.8 \%$ de quienes se autolesionan lo hacen con un solo método, el $44.9 \%$ reportaron que usan entre 2 y 4 de los métodos, el 17\% indicaron usar entre 5 y 7 de los métodos, mientras que el $5.4 \%$ reportaron usar entre 8 y 10 formas de autolesión. En relación con la función de la conducta autolesiva, en la Tabla 1 se presentan los motivos y el porcentaje para la muestra de estudio. Como se observa en la Tabla 1, los adolescentes indicaron usar la autolesión principalmente como fuente de refuerzo negativo al sustituir emociones desagradables y reducir el malestar. Así mismo, se identificó que un porcentaje importante de jóvenes informaron que la autolesión representa una forma de expresar insatisfacción con sí mismos o de distraer pensamientos acerca del suicidio. Se puede apreciar que la autolesión tiene un componente centrado en las relaciones sociales, en donde el adolescente percibe que al autolesionarse aumenta el interés y el cuidado por parte de las personas cercanas.

Tabla 1. Porcentaje de la función de la conducta autolesiva informada por los adolescentes $(n=1.517)$

\begin{tabular}{lclc}
\hline \multicolumn{1}{c}{ Función } & Porcentaje & \multicolumn{1}{c}{ Función } & Porcentaje \\
\hline Calmarse & 55.5 & Recibir atención de la familia & 21.2 \\
Reducir emociones negativas & 33.9 & Recibir cuidado de otros & 20.8 \\
Expresar rabia hacia sí mismo & 29.4 & Encajar con otros & 20.1 \\
Evadir impulso de suicidio & 27.7 & Entretención propia o de otros & 17.9 \\
Sentir una emoción fuerte & 26.9 & Castigarse & 14.9 \\
Como signo de amistad & 24.0 & Por venganza & 11.7 \\
Busca que alguien regrese & 22.8 & Lastimar a alguien cercano & 7.6 \\
\hline
\end{tabular}


Los resultados de las correlaciones entre la conducta autolesiva y las variables familiares y personales de riesgo y protección se presentan en la Tabla 2. La Tabla 2 sugiere que la conducta autolesiva aumenta de forma significativa cuando los factores de riesgo aumentan. Especialmente, se identificó que la impulsividad y la bús- queda de sensaciones son los principales factores de riesgo a nivel personal de los participantes. Así mismo, la autolesión disminuye cuando el apego familiar, entendido como un factor protector, aumenta. Por el contrario, no se identificó una asociación entre las habilidades sociales o la edad con el comportamiento autolesivo.

Tabla 2. Correlaciones entre la conducta autolesiva y variables familiares y personales $(n=1.517)$

\begin{tabular}{|c|c|c|c|c|c|c|c|c|}
\hline & 2 & 3 & 4 & 5 & 6 & 7 & Edad & Sexo \\
\hline 1. Autolesión & $.17^{* *}$ & $-.19^{* *}$ & $.13^{* *}$ & $.13^{* *}$ & $.22^{* *}$ & -.04 & .01 & $-.09^{* *}$ \\
\hline 2. Búsqueda de sensaciones & & $-.18^{* *}$ & $.20^{* *}$ & $.21^{* *}$ & $.32^{* *}$ & $.10^{* *}$ & $.14^{* *}$ & .02 \\
\hline 3. Apego familiar & & & $-.51^{* *}$ & $-.13^{* *}$ & $-.22^{* *}$ & $.20^{* *}$ & $-.09^{* *}$ & $.07^{* *}$ \\
\hline 4. Manejo familiar pobre & & & & .01 & $.30^{* *}$ & $-.23^{* *}$ & $.12^{* *}$ & $.06^{*}$ \\
\hline 5. Conflictos familiares & & & & & $.25^{* *}$ & .02 & .05 & $-.08^{* *}$ \\
\hline 6. Impulsividad & & & & & & -.04 & $.06^{*}$ & -.02 \\
\hline 7. Habilidades sociales & & & & & & & .01 & $-.09^{* *}$ \\
\hline
\end{tabular}

$* p<.05, * * p<.01$

\section{Efecto mediador de la búsqueda de sensaciones}

Dentro de los objetivos formulados del presente trabajo se propuso un modelo de mediación para estimar los efectos directos e indirectos del apego familiar en la autolesión, tal y como se indica en la Figura 1. En este modelo la búsqueda de sensaciones se comporta como una variable mediadora de dicha relación. De acuerdo con los resultados del modelo de mediación simple propuesto, se identificó que existe un efecto directo significativo y negativo entre el apego familiar y los comporta- mientos autolesivos. Sin embargo, también se identificó un efecto indirecto a través de la mediación del factor de riesgo de búsqueda de sensaciones, controlando las variables de edad y sexo (Coef. $=-.08$, IC $95 \%=-.125 /$ $-.046, p<.05)$. Los datos sugieren una relación significativa negativa entre el apego familiar y la búsqueda de sensaciones, y una relación positiva significativa entre esta última y la conducta autolesiva.

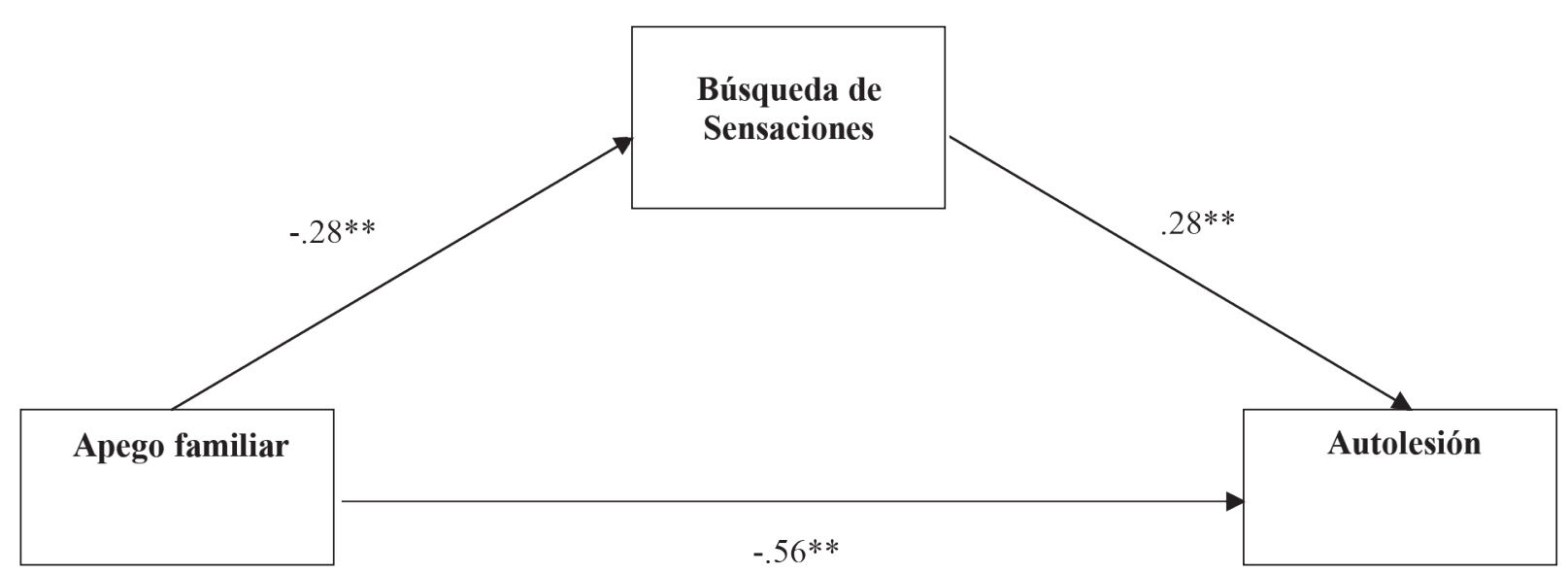

Figura 1. Diagrama sobre el efecto del apego familiar sobre la autolesión a través de la mediación de la variable búsqueda de sensaciones. Coeficienes estandarizados. Efecto indirecto, Coef. $=.08^{*}$.

${ }^{*} p<.05,{ }^{*} p<.01$ 


\section{Estudio 2}

El segundo estudio tuvo como objetivo examinar los factores personales y contextuales que se presentan de manera conjunta con la conducta autolesiva en una muestra clínica. Así mismo, como objetivo específico se buscó contrastar los resultados de la muestra no clínica (Estudio 1) con la muestra clínica del Estudio 2 con el fin de identificar posibles convergencias y divergencias dependiendo del tipo de participantes.

Las hipótesis para este estudio fuerob: (1) los pacientes que presentan conductas autolesivas deberían presentar también otros problemas psicológicos asociados (que motivan la consulta psicológica) y (2) los pacientes con autolesión presentarán afectación en los diferentes contextos de interaccón (familiar, social, laboral, personal).

\section{Método}

\section{Participantes}

Para este estudio se llevó a cabo un análisis descriptivo de 43 casos clínicos de pacientes adolescentes que reportaron comportamientos autolesivos no suicidas en un centro de atención psicológica privado al norte de Bogotá. El análisis se realizó por medio de la revisión de la historia clínica de cada paciente y como criterios de inclusión se tuvo en cuenta el reporte de compfortamientos autolesivos, el rango de edad y que hubieran autorizado el uso de la información para fines investigativos por medio del consentimiento informado.

El rango de edad de los participantes fue de 11 a 19 años, con una media de $14.5(D T=1.87)$, y el $74 \%$ fueron mujeres. Debido a la edad de los pacientes, todos reportaron como actividad ser estudiantes de educación secundaria. Frente al nivel socio-económico, el $72 \%$ se encontraban en nivel bajo, el $19 \%$ en nivel medio y el $9 \%$ en nivel alto. En cuanto a la estructura familiar, el $44 \%$ era monoparental femenina, el $42 \%$ familia con ambos padres, el 5\% de participantes se encontraban institucionalizados y el $2 \%$ correspondían a familia monoparental masculina. El 63\% de los pacientes reportaron haber recibido atención psicológica previa.

Respecto al diagnóstico principal, el 21\% de la muestra fue diagnosticado con «signos y síntomas que involucran el estado emocional», el $16 \%$ con «irritabilidad y enojo», el $12 \%$ «episodio depresivo leve» y el mismo porcentaje con «ajuste a las transiciones del ciclo vital», el $7 \%$ con «episodio depresivo moderado» y el mismo porcentaje con «problemas relacionados con el grupo primario de apoyo», el 5\% con «trastorno de la conducta» y el mismo porcentaje con «examen y obervación por razones específicas». Con un $2 \%$ se identificaron: «trastornos de la ingestión de alimentos», «tricotilomania», «trastorno opositor desafiante», «problemas relacionados con el alejamiento del hogar en la infancia», «abuso sexual», «apoyo familiar inadecuado» y «desaparición/muerte de un miembro de la familia».

Los diagnósticos secundarios de la muestra fueron: $19 \%$ «ajuste a las transiciones del ciclo vital», $12 \%$ «hechos estresantes que afectan a la familia y el hogar», $9 \%$ «síntomas y signos que involucran el estado emocional», $7 \%$ «ruptura familiar por separación o divorcio», $5 \%$ «irritabilidad y enojo». Con un 5\% se presentaron los siguientes diagnósticos: «bajo rendimiento escolar», «abuso sexual», «experiencias negativas en la infancia», «presiones inapropiadas de los padres», «problemas relacionados con la crianza del niño», «desaparición o muerte de un miembro de la familia». Con un porcentaje inferior al 2\% se presentaron: «eventos que llevaron a la pérdida de la autoestima en la infancia», «experiencias personales atemorizantes en la infancia», «apoyo familiar inadecuado», «ausencia de un miembro de la familia», «problemas especificados con el grupo primario de apoyo», «dieta y hábitos alimentarios inapropiados» $\mathrm{y}$ «acentuación de rasgos de la personalidad».

\section{Procedimiento}

El Estudio 2 tuvo un enfoque cualitativo que consistió en el análisis de las historias clínicas de pacientes adolescentes con comportamientos autolesivos a través de categorías de relevancia para la comprensión de un caso clínico.

En un primer momento, se realizó la identificación de los casos clínicos en los que hubiera un reporte de conducta autolesiva no suicida. Esta información se obtuvo de los psicólogos encargados de atender los casos, quienes brindaron a los investigadores una base de datos con los pacientes que cumplían los criterios de inclusión. Seguido, se seleccionaron los casos que autorizaron el uso de información para investigación en el consentimiento informado, lo que dio como resultado una base de datos con 43 pacientes, con los cuales se inició el análisis categórico. Las categorías para el análisis se presentan en la Tabla 3.

\section{Instrumentos}

Historia clínica. La información recogida de los participantes se obtuvo de los documentos anexos a la carpeta de historia clínica (material físico) y de los registros de atención sistematizados del centro psicológico. Principalmente, se incluyeron para el análisis los siguientes documentos: reporte de información sociodemográfica, 
Tabla 3. Operacionalización de las categorías de análisis en el Estudio 2

\begin{tabular}{ll}
\hline \multicolumn{1}{c}{ Categoría } & \multicolumn{1}{c}{ Descripción } \\
\hline Problemática que motiva la consulta & $\begin{array}{l}\text { Información inicial proveniente del paciente y/o acudiente acerca de comportamientos, cir- } \\
\text { cunstancias o eventos que resultan negativos en algún contexto. }\end{array}$ \\
$\begin{array}{l}\text { Elementos históricos relacionados } \\
\text { con el problema }\end{array}$ & $\begin{array}{l}\text { Información acerca de comportamientos, circunstancias o eventos de la historia del paciente } \\
\text { que guardan relación con la problemática identificada. }\end{array}$ \\
Sistema familiar & $\begin{array}{l}\text { Información acerca de la familia del paciente en la que se describan contextos, comporta- } \\
\text { mientos e interacciones que resulten relevantes para el caso. }\end{array}$ \\
Sistema social & $\begin{array}{l}\text { Información relevante acerca de los contextos sociales y de los comportamientos que en es- } \\
\text { tos se presentan. }\end{array}$ \\
Contexto académico & $\begin{array}{l}\text { Información relacionada con el rendimiento y la motivación escolar y los procesos de inte- } \\
\text { racción en el colegio. } \\
\text { Síntomas clínicos }\end{array}$ \\
$\begin{array}{l}\text { Información en la que se describa la presencia de comportamientos relacionados con una } \\
\text { patología clínica o de relevancia de cara a la atención psicológica. }\end{array}$ \\
$\begin{array}{l}\text { Función de la conducta autolesiva } \\
\text { Información en la que se identifique el mecanismo por medio del cual la conducta autolesiva } \\
\text { se mantiene. }\end{array}$ \\
$\begin{array}{l}\text { Información acerca de las técnicas usadas por el profesional en psicología para la interven- } \\
\text { ción del caso clínico. }\end{array}$ \\
\hline
\end{tabular}

formulaciones de caso clínico, informes psicológicos y registros de atención.

\section{Análisis estadístico}

Las categorías de análisis fueron propuestas teniendo en cuenta los componentes esenciales que la literatura indica para la comprensión de los casos clínicos (Page y Stritzke, 2006) y de acuerdo a cómo se consigna la información en la historia clínica de los pacientes (motivo de consulta, historia del problema, áreas de ajuste, conducta problema/ síntomas clínicos, análisis funcional y plan de intervención). Una vez definidas y operacionalizadas las categorías, se realizó un proceso de triangulación por parte de los investigadores para brindar mayor rigurosidad al proceso: Tres psicólogos con experiencia en el campo clínico que trabajaban en el centro de atención revisaron la información de cada paciente de manera independiente y la asignaron a una de las categorías. En un segundo momento, dicha asignación fue discutida de manera grupal y se realizaron los ajustes pertinentes hasta llegar a un consenso general.

\section{Resultados}

De acuerdo con los resultados presentados en la Tabla 4, se constata que para la mayoría de los pacientes el comportamiento autolesivo resulta la señal de alarma que motiva la remisión o la consulta. En segundo lugar, se encontró que los pacientes buscan el apoyo terapéuti- co debido a la presencia de síntomas afectivos. Así mismo, como elementos históricos, en los casos clínicos se evidencian el abandono de familiares significativos, comportamientos suicidas previos y antecedentes de abuso sexual en la infancia.

Acerca de los contextos en los que participan los pacientes se identificó que los conflictos familiares, las dificultades en las habilidades sociales, así como un bajo rendimiento académico, son las problemáticas de mayor prevalencia en los adolescentes que presentan autolesión (Tabla 5). Es de resaltar en esta categorización que las circunstancias familiares y sociales se encuentran afectadas en más de la mitad de los pacientes.

La categorización de los componentes clínicos de los casos, sugieren que los síntomas de esta muestra están centrados principalmente en el área afectiva. Adicionalmente, se identificó que la conducta autolesiva tiene como función principal para este grupo de pacientes la disminución de emociones negativas. Finalmente, la información en las historias clínicas muestra que los profesionales en psicología implementan estrategias basadas en el modelo cognitivo conductual con el objetivo de favorecer los procesos emocionales de los pacientes y en el trabajo conjunto con la familia. Estos resultados se presentan en Tabla 6.

\section{Discusión}

La conducta autolesiva no suicida representa una problemática evidente en la muestra de este trabajo. Cer- 
Tabla 4. Contenido identificado para las categorías de motivo de consulta y elementos históricos relevantes para el problema $(n=43)$

\begin{tabular}{lcc}
\hline Categorías & Frecuencia & Porcentaje \\
\hline Problemática que motiva la consulta & & 49 \\
Conducta autolesiva & 21 & 35 \\
Síntomas depresivos y/o ansiosos & 15 & 14 \\
Bajo rendimiento académico & 6 & 12 \\
Problemas de comportamiento & 5 & 9 \\
Problemas de la conducta alimentaria & 4 & 9 \\
Conflictos familiares & 4 & 7 \\
Intento suicida & 3 & 7 \\
Problemas en las relaciones sociales & 3 & 5 \\
Situación de abuso sexual & 2 & \\
Elementos históricos relacionados con el problema & & 23 \\
Abandono de un miembro de la familia & 10 & 16 \\
Intento de suicidio previo & 7 & 16 \\
Abuso sexual & 7 & 14 \\
Ruptura de relación de pareja & 6 & 12 \\
Separación de los padres & 5 & 9 \\
Antecedentes familiares de problemas emocionales & 4 & 9 \\
Fallecimiento de persona significativa & 4 & 9 \\
Historia de fracaso escolar & 4 & \\
\hline
\end{tabular}

Tabla 5. Contenido identificado para las categorías que describen los contextos familiar, social y académico de los participantes

\begin{tabular}{lcc}
\hline Categorías & Frecuencia & Porcentaje \\
\hline Sistema familiar & & \\
Conflictos familiares & 21 & 49 \\
Relación distante con alguno de los padres & 18 & 42 \\
Relación conflictiva con alguno de los padres & 16 & 37 \\
Crianza inconsistente & 15 & 35 \\
Crianza autoritaria & 7 & 16 \\
Violencia intrafamiliar & 7 & 16 \\
Relación conflictiva con hermano(s) & 7 & 16 \\
Uso castigo físico & 6 & 14 \\
Bajo involucramiento parental & 5 & 12 \\
Conflictos entre los padres & 4 & 9 \\
Sistema social & & \\
Bajas habilidades sociales & 15 & 35 \\
Conflictos interpersonales & 11 & 26 \\
Interacción con pares conflictivos & 6 & 14 \\
Insatisfacción social & 5 & 12 \\
Interacciones agresivas & 3 & 7 \\
Contexto académico & & \\
Bajo rendimiento académico & 3 & 47 \\
Víctima de intimidación escolar & 20 & 28 \\
Baja motivación escolar & 12 & 19 \\
Problemas de conducta & 8 & 16 \\
\hline
\end{tabular}


Tabla 6. Contenido identificado para las categorías clínicas de síntomas, función y tratamiento de los casos clínicos analizados

\begin{tabular}{lcc}
\hline Categorías & Frecuencia & Porcentaje \\
\hline Síntomas clínicos & & \\
Síntomas depresivos & 21 & 49 \\
Autoconcepto negativo & 18 & 42 \\
Disregulación emocional & 16 & 37 \\
Impulsividad & 15 & 35 \\
Síntomas ansiosos & 14 & 33 \\
Irritabilidad & 7 & 16 \\
Aislamiento social & 7 & 16 \\
Alteraciones de la alimentación & 5 & 12 \\
Conductas desafiantes & 5 & 12 \\
Ideas de muerte & 5 & 12 \\
Baja tolerancia a la frustración & 5 & 12 \\
Estilo evitativo de afrontamiento & 3 & 7 \\
Ideas de suicidio & 3 & 7 \\
Consumo de alcohol & 2 & 5 \\
Función de la conducta autolesiva & & \\
Refuerzo negativo por disminución & 25 & 58 \\
Refuerzo positivo por atención & 6 & 14 \\
Estrategias de intervención implementadas & & \\
Regulación emocional & 30 & 70 \\
Manejo de contingencias por cuidadores & 21 & 49 \\
Restructuración cognitiva & 15 & 35 \\
Afrontamiento & 14 & 33 \\
Solución de problemas & 12 & 28 \\
Comunicación asertiva & 11 & 26 \\
Activación conductual & 7 & 16 \\
Entrenamiento en habilidades sociales & 4 & 9 \\
\hline
\end{tabular}

ca de la mitad de la muestra no clínica del primer estudio presenta este tipo de comportamiento. Se identificó también que los adolescentes implementan una variedad de métodos para lesionarse (pellizcos, mordiscos y cortes son los principales) y que, además, los presentan frecuentemente de manera combinada, lo que sugiere que estamos frente a un patrón complejo de comportamiento problemático.

Respecto a la función de la autolesión ambos estudios sugieren que los adolescentes la llevan a cabo con el objetivo de disminuir emociones negativas. De acuerdo con la teoría, esto se explica teniendo en cuenta que la autolesión sustituye dichas emociones por sensaciones que resultan ser más tolerables que el dolor emocional (Lloyd-Richardson, Perrine, Dierker, y Kelley, 2007).

Por otro lado, Thomassin, Guérin Marion, Venasse, y Shaffer (2017) refieren una estrecha relación entre el uso de la autolesión con fines de regulación emocional y un déficit en las habilidades sociales. Al respecto, el primer estudio indicó que las habilidades sociales no representan un factor protector en los adolescentes frente a la autolesión. Sin embargo, el mismo estudio reveló que la conducta autolesiva está orientada a objetivos sociales, como, por ejemplo, percibir aceptación y pertenencia social. Este resultado podría implicar que un déficit en las habilidades sociales es un factor de riesgo para el comportamiento autolesivo, más las habilidades sociales no disminuyen la probabilidad de que este comportamiento se presente. Como soporte a esta tesis, el Estudio 2 mostró que un alto porcentaje de la muestra clínica presenta un déficit en habilidades sociales. Esto es relevante si se tiene en cuenta que dichas habilidades favorecerían una expresión emocional más adaptativa. Es importante profundizar en las características de esta relación en estudios posteriores.

A pesar de que estudios previos indican que hay una mayor prevalencia de comportamientos autolesivos en mujeres, el Estudio 1 no arrojó una correlación signifi- 
cativa entre estas dos variables. Sin embrago, en el Estudio 2 sí se observó una mayor tendencia de las mujeres a reportar conductas de este tipo dentro del proceso terapéutico. Esto alienta a realizar futuros estudios que favorezcan conclusiones más claras acerca del sexo como una variable relevante en la conducta autolesiva.

Los dos estudios permiten concluir que el ambiente familiar constituye un factor determinante para el desarrollo y mantenimiento de este tipo de comportamientos. En este sentido, el Estudio 1 permitió identificar el apego familiar como un factor protector ante la autolesión, así como la existencia de una relación directa y positiva entre los conflictos y el pobre manejo familiar, por un lado, y la decisión del adolescente de autolesionarse, por otro. Este dato se corrobora con el Estudio 2, en donde un alto porcentaje de pacientes indicó la presencia de problemáticas relevantes en el contexto familiar. Sin embargo, el modelo de mediación examinado sugiere que existen interacciones complejas entre el ambiente familiar y otras variables personales del adolescente que también hay que tener en cuenta. En concreto, se identificó que, además de darse una relación directa entre el apego familiar y la autolesión, la búsqueda de sensaciones puede constituir otra variable importante que media entre los factores contextuales y las conductas autolesivas.

El Estudio 2 muestra que la afectación emocional en pacientes que se autolesionan es el principal motivo para iniciar procesos terapéuticos. Se conoce que los síntomas afectivos resultan altamente molestos para quienes los padecen, además de generar preocupación o rechazo en las personas cercanas (Turner, Wakefield, Gratz, y Chapman, 2017). De manera específica, en este estudio se identificó que los pacientes presentaron síntomas depresivos y ansiosos por lo cual los terapeutas implementaron principalmente estrategias orientadas a fortalecer la regulación emocional en los adolescentes. Así mismo, en el grupo de pacientes se encontró coincidencia con lo reportado en estudios previos en donde los antecedentes de abuso sexual en la infancia incrementan el riesgo de presentar conductas autolesivas (Yates, 2009).

En relación con el contexto académico, los hallazgos del Estudio 2 indican que un número importante de pacientes que se autolesionan informan de un bajo rendimiento académico, así como ser víctimas de acoso o intimidación escolar. Al respecto, diferentes estudios plantean que estas dificultades con los iguales pueden alentar comportamientos autolesivos (Gulbas, Hausmann-Stabile, De Luca, Tyler, y Zayas, 2015). Otros estudios muestran que la autolesión puede aumentar la probabilidad de tener problemas a nivel social en el contexto escolar (Tang et al., 2013), lo que a su vez puede tener un impacto en el desempeño académico. Para iden- tificar la cronología con la que estas dos problemáticas se presentan será necesario desarrollar estudios longitudinales o entrevistas en profundidad con una muestra clínica.

De acuerdo con los hallazgos de este trabajo, se considera relevante seguir realizando estudios empíricos y teóricos que faciliten el reconocimiento de otros factores de riesgo y variables asociadas a la aparición, evolución y mantenimiento de esta problemática. Particularmente, se sugiere realizar estudios de causalidad con las variables más relevantes identificadas (impulsividad, búsqueda de sensaciones y dinámica familiar) para determinar su posible papel en la etiología de la conducta autolesiva. De igual manera, se considera necesario examinar otras áreas que podrían estar implicadas, tales como la percepción y actitud de los cuidadores de adolescentes con conducta autolesiva recurrente, la posible correlación de la autolesión con otras variables específicas como habilidades de expresión emocional y solución de problemas, y la identificación de variables clínicas más relevantes para la adherencia, evaluación e intervención terapéutica de esta problemática.

Como limitaciones de la presente investigación caben mencionarse el reducido tamaño de la muestra del Estudio 2 que dificulta la generalización de los resultados obtenidos y limita su validez. En la misma línea, el uso del análisis categórico en este estudio restringió posibles análisis de significanción estadística lo que suguiere la necesidad de implementar metodologías mixtas con muestras clínicas. Finalmente, dentro del análisis de historias clínicas, los registros e informes presentaron una alta variabilidad según caso y terapeuta, aumentando el riesgo de datos perdidos según variables específicas.

\section{Conflictos de intereses}

Los autores declaran que no existen conflictos de intereses.

\section{Referencias}

Adrian, M., Zeman, J., Erdley, C., Lisa, L., \& Sim, L. (2011). Emotional dysregulation and interpersonal difficulties as risk factors for nonsuicidal self-injury in adolescent girls. Journal of Abnormal Child Psychology 39, 389-400.

Arthur, M., Hawkins, J., Pollard, J., Catalano, R., \& Baglioni, A. (2002). Measuring risk and protective factors for substance use, delinquency, and other adolescent problem behaviors. The communities that care youth survey. Evaluation Review, 26, 575-6.

Baetens, I., Claes, L., Onghena, P., Grietens, H., Van Leeuwen, K., Pieters, C., ... \& Griffith, J. (2014). Nonsuicidal self-injury in 
adolescence: A longitudinal study of the relationship between NSSI, Psychological distress and perceived parenting. Journal of Adolescence, 37, 817-826.

Beckman, K., Mittendorfer-Rutz, E., Linchtenstein, P., Larsson, H., Almqvist, C., Runeson, B., \& Dahlin, M. (2016). Mental illness and suicide after self-harm among young adults: Longterm follow-up of self-harm patients, admitted to hospital care, in a national cohort. Psychological Medicine, 46, 33973405 .

Bresin, K., Carter, D. L., \& Gordon, K. H. (2013). The relationship between trait impulsivity, negative affective states, and urge for nonsuicidal self-injury: A daily diary study. Psychiatry Research 205, 227-231.

Brunner, R., Kaess, M., Parzer, P., Fischer, G., Carli, V., Hoven, C., ... \& Meszaros, G., (2014). Life-time prevalence and psychological correlates of adolescent direct self-injurious behavior: A comparative study of findings in 11 European countries. Journal of Child Psychology \& Pysychiatry, 55, 337-348.

Cyders, M. A., Coskunpinar, A., \& VanderVeen, J. D. (2016). Urgency: A common transdiagnostic endophenotype for maladaptive risk taking. In Zeigler-Hill, V., \& Marcus, D.K. (Eds). The dark side of personality: Science and practice in social, personality, and clinical psychology (pp.157-188). Washington, DC, US: American Psychological Association

Di Pierro, R., Sarno, I., Perego, S., Gallucci, M., \& Madeddu, F. (2012). Adolescent nonsuicidal self-injury: The effects of personality traits, family relationships and maltreatment on the presence and severity of behaviours. European Child \& Adolescent Psychiatry, 21, 511-520.

Global Burden of Disease Study. (2010). Global Burden of Disease Study 2010 (GBD 2010): Results by Cause 1990-2010. Seattle, US: Institute for Health Metrics and Evaluation (IHME).

Gulbas, L. E., Hausmann-Stabile, C., De Luca, S. M., Tyler, T. R., \& Zayas, L. H. (2015). An exploratory study of non-suicidal self-injury and suicidal behaviors in adolescent Latinas. American Journal of Orthopsychiatry, 85, 302-314.

Hayes, A. F. (2017). Introduction to mediation, moderation, and conditional process analysis: A regression-based approach. New York, NY: Guilford Publications.

Hilt, L. M., Nock, M. K., Lloyd-Richardson, E. E., \& Prinstein, M. J. (2008). Longitudinal study of nonsuicidal self-injury among young adolescents: Rates, correlates, and preliminary test of an interpersonal model. The Journal of Early Adolescence, $28,455-469$.

Instituto Nacional de Medicina Legal y Ciencias Forenses. (2016). Comportamiento del suicidio. Colombia 2015: violencia autoinfringida desde un enfoque forense. Medellín, Antioquia, CO: Instituto Nacional de Medicina Legal y Ciencias Forenses

Ipiña, M. J., Molina, L., y Reyna, C. (2010). Estructura factorial y consistencia interna de la escala MESSY (versión docente) en una muestra de niños argentinos. Suma Psicológica 17, 151-161.

Klonsky, E. D. (2007). The functions of deliberate self-injury: A review of the evidence. Clinical Psychology, 27, 226-239.

Klonsky, E. D., \& Glenn, C. R. (2009). Assessing the functions of non-suicidal self-injury: Psychometric properties of the Inventory of Statements About Self-injury (ISAS). Journal of Psychopathology and Behavioral Assessment, 31, 215-219.
Klonsky, E. D., \& Olino, T. M. (2008). Identifying clinically distinct subgroups of self-injurers among young adults: a latent class analysis. Journal of Consulting and Clinical Psychology, 76(1), 22.

Lin, M., You, J., Ren, Y., Wu, J., Hu, W., Yen, C., \& Zhang, X. (2017). Prevalence of nonsuicidal self-injury and its risk and protective factors among adolescents in Taiwan. Psychiatry Research, 255, 119-127.

Lloyd-Richardson, E. E., Perrine, N., Dierker, L., \& Kelley, M. L. (2007). Characteristics and functions of non-suicidal self-injury in a community sample of adolescents. Psychological Medicine, 37, 1183-1192.

Martin, J., Bureau, J. F., Yurkowski, K., Fournier, T. R., Lafontaine, M. F., \& Cloutier, P. (2016). Family-based risk factors for non-suicidal self-injury: Considering influences of maltreatment, adverse family-life experiences, and parent-child relational risk. Journal of Adolescence, 49, 170-180.

Matson, J. L., Neal, D., Fodstad, J. C., Hess, J. A., Mahan, S., \& Rivet, T. T. (2010). Reliability and validity of the Matson Evaluation of Social Skills with Youngsters. Behavior Modification, 34, 539-558.

Matson, J. L., Rotatori, A. F., \& Helsel, W. J. (1983). Development of a rating scale to measure social skills in children: The Matson Evaluation of Social Skills with Youngsters (MESSY). Behaviour Research and Therapy, 21, 335-340.

Mollà, L., Vila, S. B., Treen, D., López, J., Sanz, N., Martín, L. M., ... y Bulbena, A. (2015). Autolesiones no suicidas en adolescentes: Revisión de los tratamientos psicológicos. Revista de Psicopatología y Psicología Clínica, 20, 51-61.

Muehlenkamp, J. J., Claes, L., Havertape, L., \& Plener, P. L. (2012). International prevalence of adolescent non-suicidal self-injury and deliberate self-harm. Child and Adolescent Psychiatry and Mental Health, 6, 1-10. d

Nock, M. K., \& Favazza, A. R. (2009). Nonsuicidal self-injury: Definition and classification. In M.K. Nock, (Ed.). Understanding Nonsuicidal Self-Injury: Origins, Assessment, and Treatment. (pp.9-18). Washington, DC: American Psychological Association.

Organización Panamericana de la Salud (2014). Mortalidad por suicidio en las Américas: Informe regional. Washington, DC: Organización Panamericana de la Salud.

Organización Mundial de la Salud - World Health Organization. (2014). Preventing suicide: A global imperative. Luxembourg: World Health Organization.

Patton, J. H., \& Stanford, M. S. (1995). Factor structure of the Barratt impulsiveness scale. Journal of Clinical Psychology, 51, 768-774.

Page, A., \& Stritzke, W. (2006). Assesing clients. In Page, A., \& Stritzke, W. (Eds). Clinical Psychology for Trainees (pp. 2759). Cambridge, UK: Cambridge University Press.

Peters, E. M., Baetz, M., Marwaha, S., Balbuena, L., \& Bowen, R. (2016). Affective instability and impulsivity predict nonsuicidal self-injury in the general population: A longitudinal analysis. Borderline Personality Disorder and Emotion Dysregulation, 3, 1-7.

Robertson, C. D., Miskey, H., Mitchell, J., \& Nelson-Gray R. (2013). Variety of self-injury: Is the number of different methods on non-suicidal self-injury related to personality, psychopathology, or functions of self-injury. Archives of Suicide Research, 17, 33-40. 
Ross, S., \& Heath, N. (2002). A study of the frequency of self-mutilation in a community sample of adolescents. Journal of Youth and Adolescence, 31, 67-77.

Salvo, L., y Castro, A. (2013). Confiabilidad y validez de la escala de impulsividad de barratt (bis-11) en adolescentes. Revista Chilena de Neuro-Psiquiatría, 51, 245-254.

Silva, E. C., Benjet, C., García, F. J., Cárdenas, S. J., Gómez-Maqueo, M. E. L., \& Cruz, A. V. (2016). Adaptación y propiedades psicométricas del Inventory of Statements About Self-injury en estudiantes mexicanos. Acta de Investigación Psicológica, 6, 2544-2551.

Sornberger, M. J., Heath, N. L., Toste, J. R., \& McLouth, R. (2012). Nonsuicidal self injury and gender: Patterns of prevalence, methods, and locations among adolescents. Suicide and Life Threatening Behavior, 42, 266-278.

Spears, M., Montgomery, A. A., Gunnell, D., \& Araya, R. (2014). Factors associated with the development of self-harm amongst a socio-economically deprived cohort of adolescents in Santiago, Chile. Social Psychiatry and Psychiatric Epidemiology, 49, 629-637.

Tang, J., Ma, Y., Guo, Y., Ahmed, N.I., Yu, Y., \& Wang, J. (2013). Association of aggression and non-suicidal self-injury: A school-based sample of adolescents. PLuS One, 8, 1-7.

Thomassin, K., Guérin Marion, C., Venasse, M., \& Shaffer, A. (2017). Specific coping strategies moderate the link between emotion expression deficits and nonsuicidal self-injury in an inpatient sample of adolescents. Child and Adolescent Psychiatry and Mental Health, 11, 21.

Thyssen, L. S., y van Camp, I. (2014). La autolesión en América Latina. Salud Mental, 37, 153-157.
Trianes, M., Blanca, M. J., Muñoz, A., García, B., Cardelle-Elawar, M., e Infante, L. (2002). Relaciones entre evaluadores de la competencia social en preadolescentes: Profesores, iguales y autoinformes. Anales de psicología, 18, 197-214.

Trujillo, A., Obando, D., \& Trujillo, C. A. (2016). Family dynamics and alcohol and marijuana use among adolescents: The mediating role of negative emotional symptoms and sensation seeking. Addictive Behaviors, 62, 99-107.

Turner, B. J., Wakefield, M. A., Gratz, K. L., \& Chapman, A. L. (2017). Characterizing interpersonal difficulties among young adults who engage in nonsuicidal self-injury using a daily diary. Behavior Therapy, 48, 366-379.

Wilcox, H. C., Arria, A. M., Caldeira, K. M., Vincent, K. B., Pinchenvsky, G. B., \& O'Grady, K. E. (2012). Longitudinal predictors of past-year non-suicidal self-injury and motives among college students. Psychological Medicine, 42, 717726.

Yates, T. M. (2009). Developmental pathways from child maltreatment to nonsuicidal self-injury. In M. K. Nock (Ed.), Understanding nonsuicidal self-injury: Origins, assessment, and treatment (pp.117-137). Washington, DC, US: American Psychological Association.

Zetterqvist, M., Lundh, L., \& Svedin, C. (2013). A comparison of adolescents engaging in self-injurious behaviors with and without suicidal intent: Self-reported experiences of adverse life events and trauma symptoms. Journal of Youth Adolescence, 42, 1257-1272.

Zuckerman, M. (1994). Behavioral expressions and biosocial of sensation seeking. New York, NY: Cambridge University Press. 